Open J. Discret. Appl. Math., Vol. 1(2018), No. 2, pp. 01 - 09

Website: https://pisrt.org/psr-press/journals/odam/

ISSN: 2617-9687 (Online) 2617-9679 (Print)

http://dx.doi.org/10.30538/psrp-odam2018.0005

\title{
CHARACTERIZING TREES WITH MINIMAL ABC INDEX WITH COMPUTER SEARCH: A SHORT SURVEY
}

\author{
YIMING ZHENG, WENSHUI LIN, QI'AN CHEN ${ }^{1}$, LINSHAN HUANG, ZHIXI WU
}

\begin{abstract}
The atom-bond connectivity $(A B C)$ index of a graph $G=$ $(V, E)$ is defined as $A B C(G)=\sum_{v_{i} v_{j} \in E} \sqrt{\left(d_{i}+d_{j}-2\right) /\left(d_{i} d_{j}\right)}$, where $d_{i}$ denotes the degree of vertex $v_{i}$ of $G$. Due to its interesting applications in chemistry, this molecular structure descriptor has become one of the most actively studied vertex-degree-based graph invariants. Many efforts were made towards the elementary problem of characterizing tree(s) with minimal $A B C$ index, which remains open and was coined as the " $A B C$ index conundrum". Up to date, quite a few significant results have been obtained. In the course of research computer search plays a non-negligible role. In the present paper we review the state of the art of the problem. In addition we intend to demonstrate that, repeating the procedure "searching - conjecturing - proving" can be an applicable paradigm to cope with elusive problems of extremal graph characterization.
\end{abstract}

Mathematics Subject Classification: Primary: 05C07; Secondary: 05C90. Key words and phrases: Atom-bond connectivity index; trees; xxtremal graph; computer search.

\section{Introduction}

We consider non-trivial connected simple graphs only. Such a graph will be denoted by $G=(V, E)$, where $V=\left\{v_{0}, v_{1}, \cdots, v_{n-1}\right\}$ and are the vertex set and edge set of, respectively. If $v_{i} v_{j} \in E$, then $G-v_{i} v_{j}$ will denote the graph obtained from $\mathrm{G}$ by deleting the edge $v_{i} v_{j}$. If, in turn, $v_{i} v_{j} \notin E$, then $G+v_{i} v_{j}$ will denote the graph obtained from $G$ by adding the edge $v_{i} v_{j}$.

Received 16-08-2108. Accepted 10-11-2018.

1 Corresponding Author

(C) 2018 Yiming Zheng, Wenshui Lin, Qi'an Chen, Linshan Huang, Zhixi Wu. This is an open access article distributed under the Creative Commons Attribution License, which permits unrestricted use, distribution, and reproduction in any medium, provided the original work is properly cited. 
Let $P=u_{0} u_{1} \cdots u_{k-1} u_{k}$ be a path of length $k$ in graph $G$ with $k \geq 1, d\left(u_{0}\right) \geq 3$, $d\left(u_{k}\right) \neq 2$, and $d\left(u_{1}\right)=d\left(u_{2}\right)=\cdots=d\left(u_{k-1}\right)=2$. If $d\left(u_{k}\right) \geq 3$ then $P$ is said to be an internal path of $G$. If $d\left(u_{k}\right)=1$ then $P$ is said to be a pendent path of G.

Let $d_{i}=d\left(v_{i}\right)$ be the degree of $v_{i}$, and $\Delta=\Delta(G)$ the maximum degree of $G$. A vertex of degree 1 is called a pendent vertex. $\pi(G)=\left(d_{0}, d_{1}, \cdots, d_{n-1}\right)$ is called the degree sequence of $G$. Given a positive integer sequence $\pi=$ $\left(d_{0}, d_{1}, \cdots, d_{n-1}\right)$, if there exists a connected graph $G$ with $\pi(G)=\pi$, then $\pi$ is said to be a (graphic) degree sequence. In particular, if $G$ is a tree, then $\pi$ is called a tree degree sequence. Let $\mathcal{C}(\pi)=\{G \mid G$ is connected and $\pi(G)=\pi\}$, and $\mathcal{T}(\pi)=\{T \mid T$ is a tree and $\pi(T)=\pi\}$.

The $A B C$ index of graph $G=(V, E)$ is defined 11 as

$$
A B C(G)=\sum_{v_{i} v_{j} \in E} \sqrt{\left(d_{i}+d_{j}-2\right) /\left(d_{i} d_{j}\right)}
$$

This vertex-degree-based topological index turned out to be closely correlated with the heat of formation of alkanes [1, and a quantum-chemical explanation for its descriptive ability was provided in [2]. Gutman et al. 3] later confirmed that the $A B C$ index could reproduce the heat of formation with accuracy comparable to that of high-level ab initio and DFT (MP2, B3LYP) quantum chemical calculations. Recently, a probabilistic interpretation of the generalized $A B C$ index is provided by Estrada [4. Due to these applications, there is an increased interest in the mathematical properties of the $A B C$ index in the last few years (See [5- 36]).

From a mathematical point of view, the first question that needs to answer is for which graphs this index assumes minimal and maximal values. It was proved [5,6] that, the $A B C$ index of a graph strictly increases with addition of edges. Hence among $n$-vertex connected graphs, the complete graph $K_{n}$ uniquely maximizes the $A B C$ index, and a graph with minimal $A B C$ index is a tree. In [7] Furtula proved that, among $n$-vertex trees the star $S_{n}$ uniquely maximizes the $A B C$ index. Xing et al. 8 found some upper bounds for the $A B C$ index in some classes of trees. However, the problem of characterizing $n$-vertex tree(s) with minimal $A B C$ index is more elusive and remains open. In 9 Gutman et al. summarized the known results and coined the problem as the " $A B C$ index conundrum". After 9] there are quite a few significant developments, in both mathematical and computational aspects. In the course of research computer search plays a non-negligible role. Namely, the research paradigm of repeating the procedure "Searching - Conjecturing - Proving" was applied: (a) Computer search for trees with minimal $A B C$ index of order as large as possible by using their known properties; (b) Conjecture their properties based on the search results; (c) Prove or disprove the conjectures; (d) Go to (a). In the present paper, we will review the state of the art of the problem, as an update of [9]. In addition we intend to demonstrate that, the paradigm may be applicable to cope with other elusive problems of extremal graph characterization. 

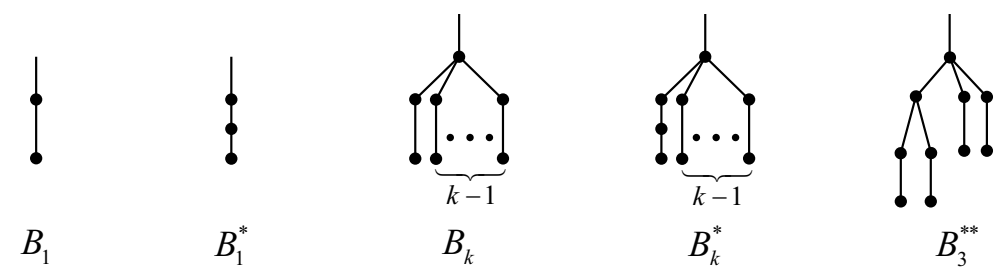

Figure 1. The branches in a min- $A B C$ tree.

For convenience, in the rest of the paper, we refer a tree with minimal $A B C$ index as a min- $A B C$ tree, and the problem of characterizing $n$-vertex tree(s) with minimal $A B C$ index as min- $A B C$ tree problem. We also assume $n \geq 10$.

\section{A brute-force and a heuristic search, and the modulo 7 conjecture}

In order to guess the general structure of min- $A B C$ trees, Furtula et al. [10] firstly conducted a brute-force computer search. Their algorithm consists of two successive steps: (1) Generating the trees recursively; (2) Computing the $A B C$ index for each generated tree and find its minimum value. Since the number of $n$-vertex trees increases rapidly with $n$, though a computer grid with 400 CPUs was employed, the computation was just performed up to $n=31$. One can refer to 11 for the search results.

Few as the search results obtained in [10, are, some structural properties of min- $A B C$ trees were observed and proved by Gutman et al. in [11].

Lemma 2.1. [12] Let $T$ be an n-vertex min- $A B C$ tree. Then

(1) $T$ has no internal paths of length $\geq 2$;

(2) $T$ has no pendent paths of length $\geq 4$;

(3) $T$ has at most one pendent path of length 3.

It was also conjectured 12 that, such a tree has no pendent paths of length 1. Soon this was confirmed by Lin et al. [13].

Lemma 2.2. [13] Let $T$ be an n-vertex min-ABC tree. Then each pendent path of $T$ is of length 2 or 3.

Lemma 2.2 reveals that, each vertex of degree 1 or 2 of an $n$-vertex min- $A B C$ tree $T$ is contained in a so-called $B_{k^{-}}$or $B_{k}^{*}$-branch (shown in Figure 1). From Lemma 2.1 (3) $T$ has a unique $B_{k}^{*}$-branch if $T$ has a pendent path of length 3. Based on these facts Gutman and Furtula 14] implicitly made the following conjecture.

Conjecture 2.3. Let $T$ be an n-vertex min-ABC tree. Then

(1) $T$ has a single high-degree vertex $v_{0}$;

(2) To $v_{0}$ only $B_{k}$ - or $B_{k}^{*}$-branches, $1 \leq k \leq 5$, are attached. 


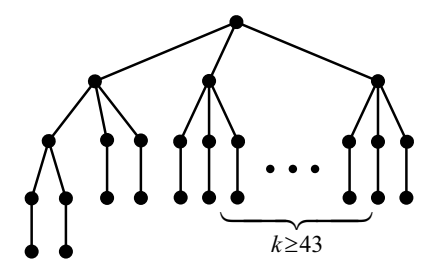

FiguRE 2. The counterexample from [15].

Actually, Conjecture 2.3 is somehow natural. Let $T$ be an $n$-vertex min- $A B C$ tree, and $T[\delta]$ the subgraph of $T$ induced by the vertices of degree at least $\delta$. From Lemma $2.1(2), T[3]$ is connected and thus is a tree. Moreover, based on the known search results at that time, $T[3]$ is conjectured to be a star (see [13]). With the priori assumptions in Conjecture 2.3, Gutman and Furtula 14 conducted a heuristic incomplete computer search for $n$-vertex min- $A B C$ tree(s) up to $n=700$. This is an easy task, because the key is to find the solution set of the Diophantine equation $n=1+2 x_{1}+5 x_{2}+7 x_{3}+9 x_{4}+11 x_{5}+x_{6}$, where $x_{i} \geq 0$ denotes the number of $B_{k}$-branches, $1 \leq i \leq 5$, and $x_{6} \in\{0,1\}$ is the number of pendent paths of length 3 .

The output of this heuristic search shows that, when $n$ is sufficiently large (e.g., $n \geq 175)$ the structure of $n$-vertex min- $A B C$ trees present a peculiar modulo 7 regularity. Therefore, the so-called "modulo 7 conjecture" was proposed [14]. Unfortunately, soon later this plausible conjecture was disproved by Ahmad et al. [15 16. In particular, the counterexample (shown in Figure 2) provided in [15] violates Conjecture 2.3 , and indicates the existence of the so-called $B_{3}^{* *}$-branches (see Figure 1) in a min- $A B C$ tree.

On the other hand, since the trees considered in [14] as candidates with minimal $A B C$ index, have an interesting structure, they were named Kragujevac trees in [17. The modulo 7 conjecture with slight corrections, was shown to be valid for Kragujevac trees.

\section{Greedy trees and search based on tree degree sequence}

Gan et al. [18, and Xing et al. [19] independently proved that, the so-called "greedy tree" minimizes the $A B C$ index in $T(\pi)$. Soon later, Lin et al. 20, generalized this result to connected graphs. One can refer to [20] for the definitions of greedy trees and BFS-graphs.

Lemma 3.1. [20] Let $\pi$ be a degree sequence. Then there exists a BFS-graph $G^{*}$ with minimal $A B C$ index in $\mathcal{C}(\pi)$.

Note that, given the degree sequence of a greedy tree (or equivalently, BFS-tree) $T, A B C(T)$ can be easily computed. With Lemma 3.1 computer search for min$A B C$ trees can be done by enumerating degree sequences of trees. Dimitrov [21] presented the first such algorithm, which consists of three successive steps. 
1. Generate all tree degree sequences (recursively);

2. Find corresponding greedy trees for each generated degree sequence;

3. Calculate the $A B C$ index of each greedy tree and select the tree with minimal value.

Comparing with the brute-force algorithm presented in [10, Dimitrov's is much more superior. It avoids generating and storing all $n$-vertex trees, and just needs to generate degree sequences of $n$-vertex trees. The search space is far less. For example, for $n=31$, there are 40,330,829,030 trees, but only 4565 tree degree sequences. Hence Dimitrov's algorithm is able to find all $n$-vertex min- $A B C$ trees for $n \leq 300$ on a single processor platform in about 15 days.

Later Dimitrov's algorithm was improved by Lin et al. 22, 23]. For convenience, we say a non-increasing positive integer sequence $\pi=\left(d_{0}, d_{1}, \cdots, d_{n-1}\right)$ is opti$\mathrm{mal}$, if it is the degree sequence of a min- $A B C$ tree. 22] and 23 obtained some features of an optimal tree degree sequence, which can be used to significantly narrow the search space. As reported in [22, for $n=31$ only 49 (about 1\%) tree degree sequences have to be generated. The MPI+OpenMP implement in [23] founds all $n$-vertex min- $A B C$ tree(s) up to $n=400$ in 23 hours on a workstation group with $36 \mathrm{CPU}$ cores. It is worth to remark that, the search results in 22] for the first time disprove Conjecture 2.3, and confirm the existence of $B_{3}^{* *}$-branches in a min- $A B C$ tree.

Based on his search result, Dimitrov modified the modulo 7 conjecture initially proposed in 14. The modified conjecture is valid for $n \leq 400$. However, this plausible conjecture was shown to be completely false for sufficiently large $n$ by Ahmadi et al. 24. Hence a much more efficient search algorithm or implementation is still desired to identify large min- $A B C$ trees.

\section{On branches and search up to order $\mathbf{1 1 0 0}$}

Let $\pi=\left(\Delta=d_{0}, d_{1}, \cdots, d_{t}, d_{t+1}, \cdots, d_{n-1}\right)\left(d_{t} \geq 3\right.$ and $\left.d_{t+1} \leq 2\right)$ be the (nonincreasing) degree sequence of a min- $A B C$ tree $T, n_{k}$ denotes the number of $k$ 's among $\left\{d_{1}, d_{2}, \cdots, d_{n-1}\right\}$, and $d=\sum_{i=1}^{t} d_{i} / t$. \# $P_{3}=(n-t-1)$ mod 2 indicates the number of paths of length 3 in $T$.

Since $B_{k}^{(*)}$-branches $\left(B_{k^{-}}\right.$or $B_{k}^{*}$-branches) are the main structure of a min- $A B C$ tree $T$, it is meaningful to pay attentions to the number $b_{k}$ of $B_{k}^{(*)}$-branches in $T$. Note please, here a $B_{3}^{* *}$-branch is regarded as one $B_{2}$-branch and two $B_{1}$-branches. In fact, in 2014 Hosseini et al. [17 have considered this task for Kragujevac trees. For general trees, most works were done by Dimitrov et al. and Du et al. in 25 30]. We summarize the main results in Theorem 4.1.

Theorem 4.1. [25-30]

(1) $b_{1} \leq 2, b_{2} \leq 11, b_{4} \leq 4$, and $b_{k}=0$ for $k \geq 5$;

(2) $b_{1} b_{4}=b_{2} b_{4}=0$;

(3) If $n>18$ and $\# P_{3}=1$, then $b_{1}=0, b_{2} \leq 2$, and $b_{k}=0$ for $k \geq 4$;

(4) If $n>415$, then $\# P_{3}=0$. 
TABLE 1. The performance of some search algorithms.

\begin{tabular}{|l|l|l|l|}
\hline Algorithm & Range of $n$ & Time & Test platform \\
\hline Brute-force search [10] & $n \leq 31$ & & PC grid, 400 CPUs \\
\hline Dimitrovs algorithm $[21]$ & $30 \leq n \leq 300$ & 15 days & PC, 2 cores, $2.3 \mathrm{GHz}$ \\
\hline Algorithm in [22] & $30 \leq n \leq 350$ & 107.8 hours & PC, 8 cores, $1.8 \mathrm{GHz}$ \\
\hline Algorithm in [23] & $30 \leq n \leq 400$ & 23 hours & PC Group, 36 cores \\
\hline Dimitrovs algorithm $[31]$ & $30 \leq n \leq 800$ & & PC, 2 cores, $2.3 \mathrm{GHz}$ \\
\hline Algorithm in [11] & $30 \leq n \leq 1100$ & 207.1 hours & PC, 4 cores, $2.2 \mathrm{GHz}$ \\
\hline
\end{tabular}

From Theorem 4.1 the features of an optimal tree degree sequence obtained in [22] and 223] were significantly refined in [11] as following.

Theorem 4.2. [11]

(1) $n_{1}=\lfloor(n-t-1) / 2\rfloor, n_{2}=\lceil(n-t-1) / 2\rceil, n_{3}=b_{2} \leq 11$, and $n_{4} \geq b_{3} \geq$ $(2 t-31) / 3$;

(2) $\# P_{3}=0$ if $n \geq 416$, and so $n_{1}=n_{2}=(n-t-1) / 2$;

(3) $(n-9) / 7 \leq t \leq(n+13) / 5$;

(4) $4 \leq \Delta \leq t$ and $\Delta \leq n / 7+3$ if $n \geq 40$;

(5) $2 \Delta+5 t \leq n+21$;

(6) $4-77 /(n-9) \leq d<5$;

By applying Theorem 4.1 Dimitrov 31 conducted a computer search for $n$ vertex min- $A B C$ tree(s) up to $n=800$. Soon later by Theorem 4.2 Lin et al. [11] presented the fastest algorithm so far. The test was performed up to $n=1100$ within 9 days on a single PC. Table 1 shows the performance of the main computer search algorithms.

\section{Further discussions}

As pointed out in [11] that, the fastest algorithm is not yet polynomial time one, and still too powerless for large $n$ (e.g., $n=5000$ ), even a large workstation group is involved. Hence towards the complete solution of the min- $A B C$ tree problem, at this point the main task is to find more properties of (sufficiently large) min- $A B C$ trees. The following are some possible directions in further investigation.

(1) Refine the upper bounds of $b_{1}, b_{2}$, and $b_{4}$. We guess $b_{1}=b_{2}=0$ and $b_{4} \leq 2$.

(2) Obtain a non-trivial lower bound of $\Delta$ on $n$ and/or $t$.

(3) Investigate the behavior of $d=\sum_{i=1}^{t} d_{i} / t$ with $n$ increases, so as to get better bounds of $d$ for large $n$. Better bounds of $d$ can help us refine Theorem 4.2. On the other hand, $d<5$ implies that, the number of high-degree vertices should be small. In fact, we guess the high-degree vertices induce a star.

\section{Acknowledgments}


This work is supported by the National Natural Science Foundation of China (No. 11771362 ).

\section{Competing Interests}

The authors declare that they have no competing interests.

\section{REFERENCES}

1. Estrada, E., Torres, L., Rodriguez, L., \& Gutman, I. (1998). An atom-bond connectivity index: modelling the enthalpy of formation of alkanes. Indian journal of chemistry. Sect. A: Inorganic, physical, theoretical \&S analytical, 37(10), 849-855.

2. Estrada, E. (2008). Atombond connectivity and the energetic of branched alkanes. Chemical Physics Letters, 463(4-6), 422-425.

3. Gutman, I., Tošović, J., Radenković, S., \& Marković, S. (2012). On atom-bond connectivity index and its chemical applicability. Indian J. Chem., 51A, 690-694.

4. Estrada, E. (2017). The ABC matrix. Journal of Mathematical Chemistry, 55(4), 10211033.

5. Chen, J., \& Guo, X. (2011). Extreme atom-bond connectivity index of graphs. MATCH Commun. Math. Comput. Chem, 65(3), 713-722.

6. Das, K. C., Gutman, I., \& Furtula, B. (2011). On atom-bond connectivity index. Chemical Physics Letters, 511(4-6), 452-454.

7. Furtula, B., Graovac, A., \& Vukičević, D. (2009). Atombond connectivity index of trees. Discrete Applied Mathematics, 157(13), 2828-2835.

8. Xing, R., Zhou, B., \& Du, Z. (2010). Further results on atom-bond connectivity index of trees. Discrete Applied Mathematics, 158(14), 1536-1545.

9. Gutman, I., Furtula, B., Ahmadi, M. B., Hosseini, S. A., Nowbandegani, P. S., \& Zarrinderakht, M. (2013). The ABC index conundrum. Filomat, 27(6), 1075-1083.

10. Furtula, B., Gutman, I., Ivanović, M., \& Vukičević, D. (2012). Computer search for trees with minimal ABC index. Applied mathematics and computation, 219(2), 767-772.

11. Lin, W., Chen, J., Wu, Z., Dimitrov, D., \& Huang, L. (2018). Computer search for large trees with minimal ABC index. Applied Mathematics and Computation, 338, 221-230.

12. Gutman, I., Furtula, B., \& Ivanovic, M. (2012). Notes on trees with minimal atom-bond connectivity index. MATCH Commun. Math Comput. Chem, 67(2), 467-482.

13. Lin, W., Lin, X., Gao, T., \& Wu X. (2013). Proving a conjecture of Gutman concerning trees with minimal ABC index. MATCH Commun. Math. Comput. Chem., 69(20), 549557.

14. Gutman, I., \& Furtula, B. (2012). Trees with smallest atom-bond connectivity index. MATCH Commun. Math. Comput. Chem., 68(1), 131-136.

15. Ahmadi, M. B., Hosseini, S. A., \& Nowbandegani, P. S. (2013). On trees with minimal atom bond connectivity index. MATCH Commun. Math. Comput. Chem, 69, 559-563.

16. Ahmadi, M.B., Hosseini, S.A., \& Zarrinderakht M. (2013). On large trees with minimal atom-bond connectivity index. MATCH Comm. Math. Comput. Chem., 69, 565-569.

17. Hosseini, S.A., Ahmadi, M.B., \& Gutman, I. (2014). Kragujevac trees with minimal atombond connectivity index. MATCH Commun. Math. Comput. Chem., 71, 5-20.

18. Gan, L., Liu, B., \& You, Z. (2012). The ABC index of trees with given degree sequence. MATCH Commun. Math Comput. Chem., 68, 137-145.

19. Xing, R., \& Zhou, B. (2012). Extremal trees with fixed degree sequence for atom-bond connectivity index. Filomat, 26, 683-688.

20. Lin, W., Gao, T., Chen, Q., \& Lin, X. (2013). On the atom-bond connectivity index of connected graphs with a given degree sequence. MATCH Commun. Math. Comput. Chem., 69, 571-578. 
21. Dimitrov, D. (2013). Efficient computation of trees with minimal atom-bond connectivity index. Appl. Math. Comput., 224, 663-670.

22. Lin, W., Chen, J., Chen, Q. A., Gao, T., Lin, X., \& Cai, B. (2014). Fast computer search for trees with minimal ABC index based on tree degree sequences. MATCH Commun. Math. Comput. Chem, 72, 699-708.

23. Lin, W., Ma, C., Chen, Q., Chen, J., Gao, T., \& Cai, B. (2015). Parallel search trees with minimal ABC index with MPI+ OpenMP. MATCH Commun. Math. Comput. Chem., 73, 337-343.

24. Ahmadi, M.B., Dimitrov, D., Gutman, I., \& Hosseini, S.A. (2014). Disproving a conjecture on trees with minimal atom-bond connectivity index. MATCH Commun. Math. Comput. Chem., 72, 685-698.

25. Dimitrov, D. (2014). On structural properties of trees with minimal atom-bond connectivity index. Discrete Applied Mathematics, 172, 28-44.

26. Dimitrov, D. (2016). On structural properties of trees with minimal atom-bond connectivity index II: Bounds on B1-and B2-branches. Discrete Applied Mathematics, 204, 90-116.

27. Du, Z., \& da Fonseca, C. M. (2016). On a family of trees with minimal atom-bond connectivity index. Discrete Applied Mathematics, 202, 37-49.

28. Dimitrov, D., Du, Z., \& da Fonseca, C. M. (2016). On structural properties of trees with minimal atom-bond connectivity index III: Trees with pendent paths of length three. Applied Mathematics and Computation, 282, 276-290.

29. Dimitrov, D. (2017). On structural properties of trees with minimal atom-bond connectivity index IV: Solving a conjecture about the pendent paths of length three. Applied Mathematics and Computation, 313, 418-430.

30. Dimitrov, D., Du, Z., \& da Fonseca, C.M. (2018). Some forbidden combinations of branches in minimal-ABC trees. Discrete Appl. Math., 236, 165-182.

31. Dimitrov, D., \& Milosavljević, N. (2018). Efficient computation of trees with minimal atom-bond connectivity index revisited. MATCH Commun. Math. Comput. Chem., 79, 431-450.

32. Das, K.C., Elumalai, S., \& Gutman, I. (2017). On ABC index of graphs. MATCH Commun. Math. Comput. Chem., 78, 459-468.

33. Das, K.C. (2010). Atom-bond connectivity index of graphs. Discrete Appl. Math., 158, 1181-1188.

34. Xing, R., Zhou, B., \& Dong, F. (2011). On atombond connectivity index of connected graphs. Discrete Appl. Math., 159(15), 1617-1630.

35. Gan, L., Hou, H., \& Liu, B. (2011). Some results on atom-bond connectivity index of graphs. MATCH Commun. Math Comput. Chem., 66, 669-680.

36. Chen, J., Liu, J., \& Guo, X. (2012). Some upper bounds for the atom-bond connectivity index of graphs. Appl. Math. Lett., 25, 1077-1081.

\section{Yiming Zheng}

School of Information Science and Engineering, Xiamen University, Xiamen 361005, China. email: 451573076@qq.com

\section{Wenshui Lin}

Fujian Key Laboratory of Sensing and Computing for Smart City, Xiamen 361005, China and School of Information Science and Engineering, Xiamen University, Xiamen 361005, China.

email: wslin@xmu.edu.cn

\section{Qi'an Chen}

School of Information Science and Engineering, Xiamen University, Xiamen 361005, China. email: cheer@xmu.edu.cn 


\section{Linshan Huang}

School of Information Science and Engineering, Xiamen University, Xiamen 361005, China. email: 574986960@qq.com

Zhixi Wu

School of Information Science and Engineering, Xiamen University, Xiamen 361005, China. email: $408763524 @ q q . c o m$ 